\title{
A relationship between the many-body theory of inelastic scattering and the distorted wave approximation $\dagger$
}

\author{
T N Rescigno, C W McCurdy Jr and V McKoy \\ Arthur Amos Noyes Laboratory of Chemical Physics, $\ddagger$ California Institute of Technology, \\ Pasadena, California 91109, USA
}

Received 18 June 1974

\begin{abstract}
In this paper we show that the first-order results of the recent many-body theory of inelastic electron scattering (Csanak, Taylor and Yaris) can be derived quite simply by a direct application of the distorted-wave and random phase approximations to the usual expression for the inelastic scattering amplitude. The result is derived both in the second quantized formalism and by the standard application of the distorted-wave approximation coupled with the random phase approximation (RPA). The RPA (or time-dependent HartreeFock theory) just provides the transition density between the initial and inelastically excited states. Possible generalizations of the procedures are discussed.
\end{abstract}

\section{Introduction}

In a series of recent papers, Taylor and coworkers have developed a theory for describing elastic (Schneider et al 1970) and inelastic (Csanak et al 1971, 1973) electron scattering based upon the Martin-Schwinger Green function technique (Martin and Schwinger 1959). The elastic theory has already been applied to electron-helium scattering with great success (Yarlagadda et al 1973) and their preliminary results on several inelastic transitions in helium give differential cross sections that are in close agreement with the best experimental results available (Thomas et al 1973).

It is the inelastic scattering formulation that is the subject of our concern in this paper. The inelastic formula, which has been shown to give excellent results for several transitions in helium, is the first-order result obtained from Taylor's many-body field theory. In this order, correlation between the incident electron and the target atom is ignored. In effect, the electron is inelastically scattered by a static transition potential. The somewhat surprising occurrence is the appearance, in Taylor's formula, of a continuum orbital for the scattered electron in the exit channel that is calculated in the field of the ground state.

A similar prescription for calculating inelastic $\mathrm{e}^{-}-\mathrm{He}$ cross sections has been recently used by Madison and Shelton (1973) in the context of the distorted-wave Born approximation. In this work, however, there seems to have been some ambiguity over the proper choice of continuum orbital for the final state. The best results were obtained with the 'unphysical' choice of computing both initial and final state orbitals in the field of the ground state. This result agrees with the first-order theory of Taylor et al. It is still difficult to assess the correctness of this choice from Madison and Shelton's

$\uparrow$ This work was supported in part by a grant from the National Science Foundation.

$\ddagger$ Contribution No 4899 . 
work, however, because they were forced to ignore certain exchange-type orthogonality terms that appeared in their formula and because all the continuum orbitals they used were calculated over spherically averaged potentials.

Our motivation for this paper is threefold. First, we wish to show that the first-order result of Csanak et al (1971) may be derived quite simply in second quantization using the random phase approximation (RPA). It is not necessary to resort to approximate solutions of the Bethe-Salpeter equation nor to use the full machinery of the MartinSchwinger formalism to obtain this theory in lowest order.

We would like to show, secondly, that identical results may be obtained by a standard application of the distorted-wave approximation coupled with the RPA. The use of the RPA in this context has several advantages over the approach used by Madison and Shelton. On the one hand, it puts correlation effects between target electrons directly into the calculation. Furthermore, the questionable omission of certain exchange-like terms is no longer a problem since we will show that, in the RPA, all such terms cancel identically.

Finally, we will comment briefly on the possibility of extending the first-order theory presented here to include some dynamical effects of target polarization, while still retaining a distorted-wave picture of the scattering process and an RPA description of the target.

\section{Inelastic electron scattering}

\subsection{Many-body treatment}

Before deriving an expression for the matrix elements of the inelastic transition operator, it is necessary to establish some notation. The closed shell ground state and excited state wavefunctions of the $N$-electron target (with energies $E_{0}$ and $E_{f}$ ) will be denoted $\psi_{0}\left(r_{1}, r_{N}\right)$ and $\psi_{f}\left(r_{1}, r_{N}\right)$. In second quantization these become the state vectors $|0\rangle$ and $|f\rangle$. The momentum of the scattered electron in the initial and final asymptotic state will be denoted by $k_{i}$ and $k_{f}$ respectively, so that the total energy is given by

$$
E=E_{0}+\frac{1}{2} k_{i}^{2}=E_{f}+\frac{1}{2} k_{f}^{2} \text {. }
$$

We will employ the annihilation and creation operators $a_{i}$ and $a_{i}^{+}$referring to oneparticle basis states which are Hartree-Fock bound and continuum spin orbitals of the ground state of the target. Hole states will be denoted by Greek subscripts and particle states by the letters $r, s, m$ and $n$. The subscripts $i, j, k$ and $l$ will be reserved for unrestricted sums. When we consider continuum particle states we will use the notation $a\left(k^{ \pm}\right)$, the superscript on the momentum $k$ indicating outgoing or incoming wave boundary conditions. Finally second-quantized operators will always be denoted with carats ().

The Hamiltonian in second-quantized form is

$$
\hat{H}=E_{\mathrm{HF}}+: \hat{H}_{0}:+: \hat{V}:
$$

where $E_{\mathrm{HF}}$ is the Hartree-Fock ground state energy of the target, and the operators (normal ordered with respect to the Hartree-Fock particle-hole vacuum) are

$$
\begin{aligned}
& : \hat{H}_{0}:=\sum_{i} \epsilon_{i}: a_{i}^{+} a_{i}: \\
& : \hat{D}:=\frac{1}{4} \sum_{i j k l}(i j|| k l)_{\mathrm{a}}: a_{i}^{+} a_{j}^{+} a_{l} a_{k}:
\end{aligned}
$$


The antisymmetrized matrix elements are defined by

$$
(i j|| k l)_{\mathrm{a}}=(i j|| k l)-(i j|| l k)
$$

where

$$
(i j|| k l)=\int \phi_{i}^{*}\left(r_{1}\right) \phi_{j}^{*}\left(r_{2}\right) \frac{1}{\left|r_{1}-r_{2}\right|} \phi_{k}\left(r_{1}\right) \phi_{l}\left(r_{2}\right) \mathrm{d}^{3} r_{1} \mathrm{~d}^{3} r_{2} .
$$

The transition matrix element for inelastic electron scattering with the target initially in its ground state $|0\rangle$ and with final state $|f\rangle$ can be expressed as $\dagger$

$$
T_{f i}=\left\langle f\left|\left[a\left(k_{f}^{-}\right), \hat{V}_{\mathrm{res}}\right]\right| 0, k_{i}^{+}\right\rangle
$$

where $\left|0, k_{i}^{+}\right\rangle$is the exact scattering wavefunction of the $N+1$ particle system. The residual interaction $\hat{V}_{\text {res }}$ is $\hat{V}_{\text {total }}-\hat{V}_{\text {Hartree-Fock }}$ so $\hat{V}_{\text {res }}$ can be replaced by $: \hat{V}:$ to within an additive constant.

The potential $: \hat{V}:$ may be partitioned

$$
: \hat{V}:=\hat{V}_{1}+\hat{V}_{2}+\hat{V}_{3}
$$

where

$$
\begin{aligned}
& \hat{V}_{1}=\frac{1}{4} \sum_{m \alpha n \beta}(m n|| \alpha \beta)_{\mathrm{a}} a_{m}^{+} a_{\alpha} a_{n}^{+} a_{\beta}+\mathrm{hc}+\sum_{m \alpha n \beta}(m \beta|| \alpha n)_{\mathrm{a}} a_{m}^{+} a_{\alpha} a_{\beta}^{+} a_{n} \\
& \hat{V}_{2}=\frac{1}{2} \sum_{m \alpha n r}(m \alpha|| n r)_{\mathrm{a}} a_{m}^{+} a_{\alpha}^{+} a_{r} a_{n}+\mathrm{hc}+\frac{1}{2} \sum_{m \alpha \gamma \beta}(m \alpha|| \gamma \beta)_{\mathrm{a}} a_{\gamma} a_{\beta} a_{m}^{+} a_{\alpha}^{+}+\mathrm{hc} \\
& \hat{V}_{3}=\frac{1}{4} \sum_{m n r s}(m n|| r s)_{\mathrm{a}} a_{m}^{+} a_{n}^{+} a_{s} a_{r}+\frac{1}{4} \sum_{\alpha \beta \gamma \delta}(\alpha \beta|| \gamma \delta)_{\mathrm{a}} a_{\delta} a_{\gamma} a_{\alpha}^{+} a_{\beta}^{+} .
\end{aligned}
$$

Thus $\hat{V}_{1}$ is the interaction between particle-hole pairs; $\hat{V}_{2}$ describes the scattering of a particle or hole by a particle-hole pair; and $\hat{V}_{3}$ is the particle-particle and hole-hole interaction.

The final target state may be written with the aid of the excitation operator defined by (Rowe 1968)

$$
\begin{aligned}
& |f\rangle=\hat{\mathrm{O}}_{f}^{+}|0\rangle \\
& \hat{\mathrm{O}}_{f}|0\rangle=0 .
\end{aligned}
$$

We may construct an approximation to equation (5) by first choosing $\hat{\mathrm{O}}_{f}^{+}$to be the RPA excitation operator

$$
\hat{\mathrm{O}}_{f}^{+} \approx \sum_{m \alpha} Y_{m \alpha}^{(f)} a_{m}^{+} a_{\alpha}-Z_{m \alpha}^{(f)} a_{\alpha}^{+} a_{m}
$$

where $Y_{m \alpha}^{(f)}$ and $Z_{m \alpha}^{(f)}$ are amplitudes satisfying the usual RPA equations (Rowe 1968).

If we next make the lowest order approximation to $\left|0, k_{i}^{+}\right\rangle$and, consistent with the distorted wave picture, set

$$
\left|0, k_{i}^{+}\right\rangle \approx a^{+}\left(k_{i}^{+}\right)|0\rangle
$$

then equation (3) becomes

$$
T_{f i} \approx\langle 0|\left[\mathrm{O}_{f},\left[a\left(k_{f}^{-}\right),: V:\right] a^{+}\left(k_{i}^{+}\right]|0\rangle .\right.
$$

$\uparrow$ The formulation of scattering theory in the language of second quantization is standard in nuclear physics theory. For an excellent review, see Villars (1966). 
The commutators in equation (10) lower the particle rank of the operator expression, and hence we use the same approximation to evaluate equation (10) as in deriving the RPA equations of which the amplitudes specifying $\mathrm{O}_{f}^{+}$are solutions. Thus we evaluate the matrix element in equation (10) with respect to the Hartree-Fock particle-hole vacuum. It is then easy to show that the contributions to equation (10) from $\hat{V}_{1}$ and $\hat{V}_{3}$ vanish identically, as does the contribution of the part of $\hat{V}_{2}$ describing the interaction of a hole with a particle-hole pair. Repeated application of Wick's theorem results in the expression

$$
T_{f i} \approx \sum_{m \alpha}\left\{Y_{m \alpha}^{*(f)}\left(m k_{f}^{-} \| \alpha k_{i}^{+}\right)_{\mathrm{a}}+Z_{m \alpha}^{*(f)}\left(\alpha k_{f}^{-} \| m k_{i}^{-}\right)_{\mathbf{a}}\right\}
$$

This is identical to the first-order expression for inelastic scattering derived by Csanak et al (1971), and can also be written in the form of a matrix element of a 'transition potential' between two Hartree-Fock continuum orbitals.

\subsection{Distorted-wave approximation}

We now show that the result obtained in the previous section can also be found by a straightforward use of the distorted-wave approximation coupled with the RPA.

To the interaction potential $V$ between an incident electron and an $N$-electron atom or molecule, we add and subtract the operators $J-K$, the Hartree-Fock Coulomb and exchange operators for the target in its ground state. We then partition $V$ into two terms, $V=V_{\mathrm{I}}+V_{\mathrm{Il}}$, where

$$
\begin{aligned}
& V_{1} \equiv \sum_{\text {nuclei }}-\frac{Z_{n}}{r_{n}}+J-K \\
& V_{\mathrm{II}} \equiv \sum_{i=1}^{N} \frac{1}{\left|r-r_{i}\right|}-J+K .
\end{aligned}
$$

Using the well known two-potential formula (Messiah 1968), the transition matrix element for inelastic electron scattering can be written as

$$
T_{f i}=\left(\psi_{f}\left(r_{1}, r_{N}\right) \mathrm{e}^{i k_{f} r}\left|V_{\mathrm{l}}\right| \psi_{1 k_{i}}^{+}\right)+\left(\psi_{1 k_{f}}^{-}\left|V_{11}\right| \psi^{+}\right)
$$

$\psi^{+}$is the exact, antisymmetric wavefunction of the full interacting system with outgoing wave boundary conditions. $\psi_{1 k_{i}}^{+}$and $\psi_{1 k_{f}}^{-}$are scattering solutions of the operator $-\frac{1}{2} \nabla_{r}^{2}+H_{\text {targe } 1}+V_{\mathrm{I}}$. Thus we can write

$$
\begin{aligned}
& \psi_{1 k_{i}}^{+}=\psi_{0}\left(r_{1}, r_{N}\right) \phi_{k_{i}}^{+}(r) \\
& \psi_{1 k_{f}}^{-}=\psi_{f}\left(r_{1}, r_{N}\right) \phi_{k_{j}}^{-}(r) .
\end{aligned}
$$

Note that these solutions are not antisymmetric under interchange of $r$ with $\left\{\boldsymbol{r}_{i}\right\}$ since they come from a non-symmetric Hamiltonian. Thus, the first term in equation (13) is rigorously zero due to the orthogonality of $\psi_{0}$ and $\psi_{f}$. Equation (13) is exact; we now make the distorted-wave approximation and set $\psi^{+}$equal to an antisymmetrized product of $\psi_{0}\left(r_{1}, r_{N}\right)$ and $\phi_{k_{i}}^{+}(r)$, giving

$$
T_{f i} \sim\left\{\psi_{f}\left(r_{1}, r_{N}\right) \phi_{k_{f}}^{-}(r)\left|V_{\mathrm{ll}}\right| \mathscr{A}\left[\psi_{0}\left(r_{1}, r_{N}\right) \phi_{k_{i}}^{+}(r)\right]\right\}
$$


Without too much trouble, it can be shown that equation (15), which now contains direct and exchange contributions, can be conveniently grouped into two terms:

$$
\begin{aligned}
T_{f i}=N\left(\psi_{f}\left(r_{1},, r_{N}\right) \phi_{k_{f}}^{-}(r) \frac{1}{\left|r-r_{1}\right|} \Psi_{0}\left(r_{1}, r_{N}\right) \phi_{k_{i}}^{+}(r)-\Psi_{0}\left(r, r_{N}\right) \phi_{k_{i}}^{+}\left(r_{1}\right)\right) \\
-N\left(\psi_{f}\left(r_{1}, r_{N}\right) \phi_{k_{f}}^{-}(r)\left|\sum_{i \neq 1} \frac{1}{\left|r-r_{i}\right|}-J+K\right| \phi_{k_{i}}^{+}\left(r_{1}\right) \Psi_{0}\left(r, r_{N}\right)\right) .
\end{aligned}
$$

We now show that, in the RPA, the first term above gives the same result obtained previously and that the second term vanishes. In order to demonstrate this, the matrix elements in (16) must be rewritten in second-quantized form and hence permutation operators will be introduced so that the coordinates of the wavefunctions appear in identical order on both sides of the matrix elements.

For example, the first term in equation (16) can be expressed as

$$
N\left(\psi_{f}\left(r_{1}, r_{N}\right) \phi_{k_{f}}^{-}(r)\left|\frac{1}{\left|r-r_{1}\right|}\left(1-P_{r, r_{1}}\right)\right| \Psi_{0}\left(r_{1}, r_{N}\right) \phi_{k_{i}}^{+}(r)\right)=\sum_{i j}\left\langle f\left|a_{i}^{+} a_{j}\right| 0\right\rangle u_{i j}
$$

where

$$
u_{i j}=\left(i k_{f}^{-} \| j k_{i}^{+}\right)_{\mathbf{a}}
$$

and $P_{r, r_{1}}$ interchanges coordinates $r$ and $r_{1}$. Employing the same strategy used in the previous section, we write this as

$$
\left\langle 0\left|\left[\mathrm{O}_{f}, \hat{O}\right]\right| 0\right\rangle
$$

which, in the RPA, becomes

$$
\sum_{m, \alpha} u_{m \alpha} Y_{m \alpha}^{*(f)}+u_{\alpha m} Z_{m \alpha}^{*(f)}
$$

coinciding with the expression derived earlier.

Finally, we consider the second part of (16). The term involving $-J+K$ can again be written in second quantization as the matrix element of a one-electron operator,

$$
-N\left(\psi_{f}\left(r_{1}, r_{N}\right) \phi_{k_{f}}^{-}(r)\left|(-J+K) P_{r, r_{1}}\right| \phi_{k_{i}}^{+}(r) \Psi_{0}\left(r_{1}, r_{N}\right)\right)=\sum_{i j}\left\langle f\left|a_{i}^{+} a_{j}\right| 0\right\rangle w_{i j}(18)
$$

where

In the RPA, this becomes

$$
w_{i j}=\delta_{i, k_{i}^{+}} \sum_{u}\left(k_{f}^{-} u|| j u\right)_{\mathbf{a}} .
$$

$$
\sum_{\alpha, u} Y_{k_{i}^{+}, \alpha}^{*(f)}\left(k_{f}^{-} u \| \mid \alpha u\right)_{\mathbf{a}} .
$$

In similar fashion, the term involving $\Sigma_{i \neq 1} 1 /\left|r-r_{1}\right|$ can be written as the matrix element of a two-electron operator:

with

$$
-\sum_{i j k l}\left\langle f\left|a_{i}^{+} a_{j}^{+} a_{k} a_{l}\right| 0\right\rangle V_{i j l k}
$$

$$
V_{i j l k}=\delta_{i, k_{i}^{+}}\left(k_{f}^{-} j|| l k\right)
$$

which, in the RPA, becomes

$$
-\sum_{i j k l m \alpha}\left\langle 0\left|\left[Y_{m \alpha}^{*} a_{\alpha}^{+} a_{m}-Z_{m \alpha}^{*} a_{m}^{+} a_{\alpha}, a_{i}^{+} a_{j}^{+} a_{k} a_{l}\right]\right| 0\right\rangle V_{i j l k} .
$$


Straightforward evaluation of this expression gives

$$
-\sum_{\alpha u} Y_{k_{i}^{+}, \alpha}^{*}\left(k_{f}^{-} u \| \alpha u\right)
$$

which cancels the term in (18) identically, giving the same expression for $T_{f i}$ derived in the previous section.

\section{Discussion}

We have shown that a many-body description of inelastic electron-scattering produces, in lowest order, a distorted-wave formula in which the scattered electron appears, both initially and finally, in continuum Hartree-Fock orbitals generated in the field of the ground state. This picture, when coupled with an RPA description of the target, gives a simple and unambiguous prescription for calculating inelastic transition matrix elements. Such a picture would be expected to incorporate the most important effects in non-resonant scattering.

We should like to point out that it would be possible to incorporate some polarization effects into the formalism and still retain the distorted-wave picture. For example, one could go through the analysis of the previous section with an elastic optical potential as the distorting potential in place of the Hartree-Fock operators. The optical potential is identical to the proper self-energy of many-body theory (Bell and Squires 1959) and could be approximated in a variety of ways (Fetter and Watson 1965, Schneider et al 1970, Rosenberg and Tolchin 1973). The continuum orbitals of equation (14) would become optical model wavefunctions which satisfy a one-particle Lippmann-Schwinger equation with the proper self-energy as the potential. Even though the leading term in the self-energy is the Hartree-Fock potential, the counterpart of equation (11) would be different as a result of the fact that the continuum orbitals are no longer part of the orthonormal set of Hartree-Fock orbitals used to expand the excitation operator $\mathrm{O}_{f}^{-}$. In addition, new contributions to $T_{f i}$ would be made by the energy-dependent part of the optical potential. An attractive feature of this scheme is that it avoids the difficulties of computing continuum orbitals in the field of the excited state. For example, we could make use of the fact that the optical potential for a closed shell, homonuclear diatomic molecule has simple cylindrical symmetry, whereas the potential of an arbitrary excited state would not.

Although the effects of polarization would be included to some extent in the approach outlined above, final state interaction effects would not be completely accounted for. However it is well known that an exact formulation of inelastic scattering can be made in which the transition matrix retains the form of the distorted-wave expression, ie a matrix element between elastic optical model continuum orbitals of an energy-dependent transition potential (Gross 1969, Emrich 1971, Csanak et al 1971). In the exact theory the transition potential may no longer be constructed as simply as in the distorted-wave picture, but instead requires the solution of the Bethe-Salpeter equation of many-body theory.

\section{References}

Bell J S and Squires E J 1959 Phys. Rev. Lett. 3 96-7

Csanak Gy, Taylor H S and Tripathy D N 1973 J. Phys. B: Atom. Molec. Phys. $62040-54$ 
Csanak Gy, Taylor H S and Yaris R 1971 Phys, Rev, A 3 1322-8

Emrich K 1971 Nucl. Phys. A $1601-24$

Fetter A L and Watson K M 1965 Advances in Theoretical Physics vol 1 ed K A Brueckner (New York: Academic Press) 115-94

Gross D 1969 Nucl. Phys. A $12945-63$

Madison D H and Shelton W N 1973 Phys. Rev. A $7499-513$

Martin P C and Schwinger J S 1959 Phys. Rev. 115 1342-73

Messiah A 1968 Quantum Mechanics (Amsterdam: North Holland) 839

Rosenberg L and Tolchin S 1973 Phys. Rev. A 8 1702-9

Rowe D J 1968 Rev. Mod. Phys. $40153-66$

Schneider B, Taylor H S and Yaris R 1970 Phys. Rev. A 1855

Thomas L D, Yarlagadda B S, Csanak Gy and Taylor H S 1973 Comp. Phys. Comm. 6 $316-30$

Villars F 1966 Fundamentals in Nuclear Theory eds A de-ShaLit and C Villi (Vienna; IAEA) 269-331

Yarlagadda B S, Csanak Gy, Taylor H S, Schneider B and Yaris R 1973 Phys. Rev. A 7 146-54 